\title{
Nonlinear interfaces: intrinsically nonparaxial regimes and effects
}

\author{
Julio Sánchez-Curto ${ }^{1}$, Pedro Chamorro-Posada ${ }^{1}$ and Graham \\ S. McDonald ${ }^{2}$ \\ 1 Departamento de Teoría de la Señal y Comunicaciones e Ingeniería Telemática, \\ Universidad de Valladolid, ETSI Telecomunicación, Campus Miguel Delibes s/n, \\ Valladolid 47011, Spain \\ 2 Joule Physics Laboratory, School of Computing, Science and Engineering, \\ University of Salford, Salford M5 4WT, UK
}

\begin{abstract}
The behaviour of optical solitons at planar nonlinear boundaries is a problem rich in intrinsically nonparaxial regimes that cannot be fully addressed by theories based on the nonlinear Schrödinger equation. For instance, large propagation angles are typically involved in external refraction at interfaces. Using a recently proposed generalised Snell's law for Helmholtz solitons, we analyse two such effects: nonlinear external refraction and total internal reflection at interfaces where internal and external refraction, respectively, would be found in the absence of nonlinearity. The solutions obtained from the full numerical integration of the nonlinear Helmholtz equation show excellent agreement with the theoretical predictions.
\end{abstract}

PACS numbers: 42.65.Tg, 42.81.Dp 


\section{Introduction}

Interfaces have played a crucial role within electromagnetic theory, since they constitute one of the most common problems in the description of propagation phenomena. The field of nonlinear optics is not an exception and during the last few decades there have been various contributions to the understanding of the evolution of wave packets or beams at a planar boundary separating nonlinear media. The first studies of nonlinear interfaces are dated between the late 70's and the early 80's and were restricted to Gaussian beams at the boundary between a linear and a nonlinear (Kerr-type) medium $[1,2,3,4]$. In the transition from the 80's to the 90's, a second approach focused on interfaces between two Kerr-type nonlinear media $[5,6,7,8,9,10]$. The particlelike model $[6,7,8,9]$ was then introduced, where a soliton is represented as a quasiparticle which evolves according a potential defined by the nature of the nonlinear interface. Soliton characteristics, such as transverse velocity and amplitude, were related to interface parameters to establish criteria for soliton evolution at the interface. In the middle 90s, an alternative approach to the problem was introduced in the form of an adiabatic theory $[11,12]$. This was applied to the study of the boundary between two media of quite dissimilar properties, such as linear/nonlinear interfaces. In recent years, other types of nonlinear interfaces such as in photorefractive crystals [13] and in quadratic media [14] have also been analysed.

All these earlier works have in common the description of the evolution of optical beams in terms of the Nonlinear Schrodinger (NLS) equation, where the slowly varying envelope approximation (SVEA) is assumed [15]. Nevertheless, the behaviour of solitons at nonlinear interfaces may lie beyond the limits of the paraxial approximation, for instance, when external refraction occurs at the interface. In such scenarios, a full nonparaxial analysis is needed to retain the full angular extent of the problem and thus overcome the limitations of paraxial analyses. Nonparaxiality is often a misunderstood term, because it can refer to different contexts of distinct nonparaxial character: highintensity and, separately, large angles of propagation.

The first type of nonparaxiality arises in the evolution of ultra-narrow beams in nonlinear media. The suitability of the NLS for describing the evolution of such beams was questioned by Akhmediev et al. [16], who uncovered limitations of the NLS in scenarios of strong focusing. For ultra-narrow beams, a full vectorial analysis starting from the Maxwell equations can also be necessary [17, 18, 19] to include the tensorial refractive index dependence. Solutions to these equations in the form of bright [20] and dark [21, 22] nonparaxial solitons have been reported and analysed.

The second type of nonparaxiality occurs in the rapid evolution of the field envelope of a broad (when compared to the wavelength) beam propagating at a large angle to the longitudinal axis. This nonparaxiality is well described by the scalar nonlinear Helmholtz (NLH) equation $[23,24]$ which has been proposed to overcome the limitations of the NLS, for instance, by arresting soliton collapse in a focusing Kerr-type medium [23] and for which exact analytical soliton solutions have been found [24, 25, 26]. Substantial 
differences with paraxial theory are not only revealed by the exact bright Kerr soliton solutions of the NLH equation but are also found in dark Kerr [27], two-component [28], boundary [29] and bistable [30] Helmholtz soliton solutions. When the full Helmholtz approach is used, significant differences with the predictions of NLS theory are also found at a fundamental level, for example, when analysing soliton collisions [31].

As regards numerical investigations, novel methods have been developed to understand nonlinear phenomena governed by the NLH equation, where backscattered waves can accompany a forward propagating beam. Leaving aside the general framework proposed by Ferrando et al. [32] for studying the propagation of electromagnetic fields with backscattered components, two different numerical strategies for treating backward waves arising in the NLH are proposed. Fibich and Tsynkov have introduced a twoway arbitrary boundary conditions model [33, 34] that can suppress the reflection of backscattered waves without affecting the propagation conditions for the forward propagating beam. With this method, the arrest of soliton collapse for the $(2+1) \mathrm{D}$ NLH and the formation of nonparaxial solitons for the $(1+1) \mathrm{D}$ NLH have been numerically demonstrated [35]. A different numerical approach to solving the NLH is the nonparaxial beam propagation method (NBPM) [36], which combines both finite difference and spectral methods. Backscattered waves are filtered out, thus avoiding an evanescent backward field, that can appear to grow in the forward direction and hence masks the contribution of the forward propagating field. This scheme has been applied to the phenomena studied in [27, 28, 29, 30, 31].

In this paper, the NBPM is used to study the behaviour of Helmholtz solitons at nonlinear interfaces. In a previous work [37] a generalised Snell's law was introduced and its validity was assessed for interfaces exhibiting linear internal refraction, where beams crossing an interface undergo small angular deviations. The results presented in this paper extend the analysis of this generalised Snell's law (Section 2). Moreover, we also explore highly nonparaxial contexts, not previously addressed in [37] and found at interfaces exhibiting nonlinear and linear external refraction. Nonlinear external refraction is demonstrated to exist at interfaces where internal refraction would be found for linear plane waves (Section 3). For the case of interfaces admitting linear external refraction, we show that critical angles are also allowed according to the generalised Snell's law (Section 4).

\section{Generalised Snell's law for Helmholtz solitons}

The time-independent complex field envelope $E(x, z)$ of a continuous wave TE-polarised beam evolves according to a two dimensional Helmholtz equation

$$
\frac{\partial^{2} E}{\partial z^{2}}+\frac{\partial^{2} E}{\partial x^{2}}+\frac{\omega^{2}}{c^{2}} n^{2} E=0
$$

where $n$ is the nonlinear refractive index. If we consider a forward propagating beam $E(x, z)=A(x, z) e^{j k z}$ and employ the normalisations $\zeta=z / L_{D}$ and $\xi=2^{1 / 2} x / w_{0}, w_{0}$ 
being a transverse scale parameter equal to the waist of a reference Gaussian beam of diffraction length $L_{D}=k w_{0}^{2} / 2,(1)$ is transformed into

$$
\kappa \frac{\partial^{2} A}{\partial \zeta^{2}}+j \frac{\partial A}{\partial \zeta}+\frac{1}{2} \frac{\partial^{2} A}{\partial \xi^{2}}-\frac{1}{4 \kappa}\left(1-\frac{n^{2}}{n_{01}^{2}}\right) A=0
$$

In (2), $\kappa=1 / k^{2} w_{0}^{2}$ is a nonparaxiality parameter $[23,24]$ and $n_{01}$ is the linear refractive index of a first Kerr-type nonlinear material whose total refractive index is $n_{01}+\alpha_{1}|E|^{2}$ where $\alpha_{1} \ll n_{01}$. If we now include in our analysis a second Kerr-type medium with $n=n_{02}+\alpha_{2}|E|^{2}$ and consider the normalisation $A(\xi, \zeta)=\left(n_{01}{ }^{2} / k \alpha_{1} L_{D} n_{02}\right)^{1 / 2} u(\xi, \zeta)$, (2) can be rewritten as

$$
\kappa \frac{\partial^{2} u}{\partial \zeta^{2}}+j \frac{\partial u}{\partial \zeta}+\frac{1}{2} \frac{\partial^{2} u}{\partial \xi^{2}}-\left(\frac{\Delta}{4 \kappa}-\alpha|u|^{2}\right) u=0,
$$

where interface parameters relating the linear and nonlinear refractive indexes of the adjoining media, separated by a planar boundary,

$$
\Delta \equiv 1-\left(\frac{n_{02}}{n_{01}}\right)^{2}
$$

and

$$
\alpha \equiv \frac{\alpha_{2}}{\alpha_{1}}
$$

respectively, have been introduced. In absence of discontinuity, $\Delta=0$ and $\alpha=1$, one recovers the NLH for a homogeneous medium [24] from (3), which can be written as [37]

$$
\kappa \frac{\partial^{2} u}{\partial \zeta^{2}}+i \frac{\partial u}{\partial \zeta}+\frac{1}{2} \frac{\partial^{2} u}{\partial \xi^{2}}+|u|^{2} u=\left[\frac{\Delta}{4 \kappa}+(1-\alpha)|u|^{2}\right] H(\xi) u
$$

where the Heaviside function $H(\xi)$, with $H(\xi)=0$ for $\xi<0$ and $H(\xi)=1$ for $\xi \geq 0$, has been used to represent a planar boundary at $\xi=0$.

By phase-matching of exact soliton solutions [26] for $\xi<0$ and $\xi \geq 0$, one obtains a generalised Snell's law [37] that governs the evolution of beams at boundary separating two Kerr focusing media

$$
\gamma n_{01} \cos \left(\theta_{i}\right)=n_{02} \cos \left(\theta_{t}\right)
$$

$\theta_{i}$ and $\theta_{t}$ are the angles of incidence and refraction (between the beam and the interface), respectively,

$$
\gamma=\left(\frac{1+2 \kappa \eta_{0}^{2}}{1+2 \kappa \eta_{0}^{2} \alpha(1-\Delta)^{-1}}\right)^{1 / 2}
$$

is a nonlinear correction term, and $\eta_{0}$ is the incident soliton amplitude. Since $\gamma$ is responsible for all nonlinear beam corrections to the familiar linear plane-wave relationship, one can analyse (8) in terms of constant $\gamma$ lines, as shown in figure 1. Solid lines represent combinations of $\Delta$ and $\alpha$ which preserve $\gamma$ constant. The $\Delta-\alpha$ plane has been divided into four regions by means of two perpendicular dashed lines, $\Delta=0$ and $\alpha=1$, corresponding to the absence of discontinuity in the linear and nonlinear refractive indexes, respectively. 
(a)

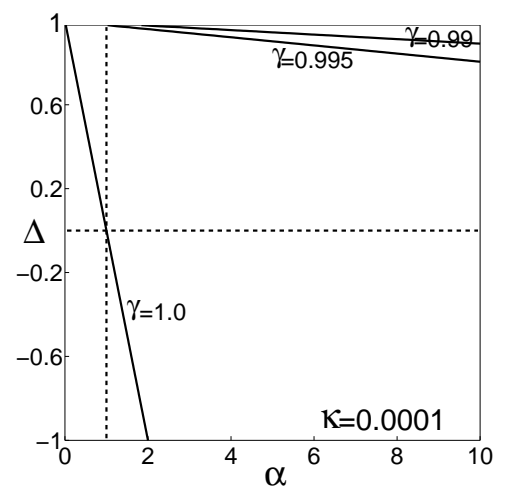

(b)

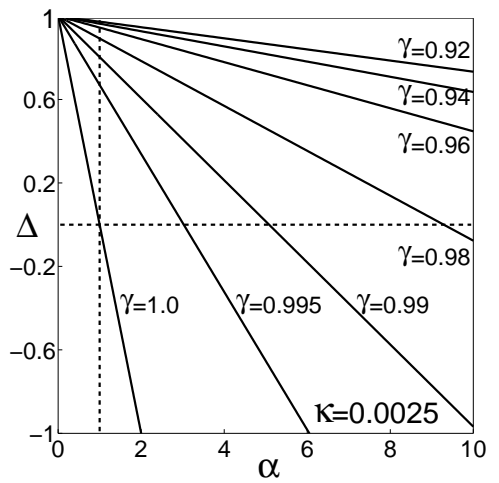

Figure 1. $\gamma$-constant curves as a function of $\Delta$ and $\alpha$ for (a) $\kappa=10^{-4}$ and (a) $\kappa=2.5 \times 10^{-3}$. In both cases, $\eta_{0}=1$.

Two completely different scenarios are found as $\kappa$ changes from $10^{-4}$ to $2.5 \times 10^{-3}$. In the first case, figure 1(a), significant nonlinear correction appear only at $\Delta \sim \eta_{0}$, since the constant $\gamma$ lines are concentrated in the $\Delta \rightarrow 1$ region. This condition is equivalent to the one obtained in previous paraxial analysis based on the NLS [6], where nonlinear corrections arose when the soliton amplitude was of the same order as $\Delta$. In contrat to this, for $\kappa=2.5 \times 10^{-3}$ and figure $1(\mathrm{~b})$, constant $\gamma$ lines are widespread over the entire $\Delta-\alpha$ plane and nonlinear corrections affect all kinds of interfaces.

From (7) and (8) one obtains an expression for the critical angle, $\theta_{c}$, defined as the smallest angle of incidence for which a transmitted soliton is found in the second medium. Setting $\theta_{t}=0$ in (7), one obtains

$$
\tan \left(\theta_{c}\right)=\left(\frac{\Delta+2 \kappa \eta_{0}^{2}(1-\alpha)}{1-\Delta+2 \kappa \eta_{0}^{2} \alpha}\right)^{1 / 2} .
$$

Figure 2(a) displays the angle of refraction of a Helmholtz soliton transmitted through a nonlinear interface with $\Delta=0.005$ and $\alpha=2$. When $\Delta \gg 2 \kappa \eta_{0}^{2}, \theta_{t}$ (solid line) has a value very close to that expected for a plane wave in the absence of nonlinearity (dashed-dotted line). As $2 \kappa \eta_{0}^{2} \sim \Delta$, the nonlinear contribution to the refractive index produces a significant modification to the angle of refraction and the generalised Snell's law predicts that nonlinear external reflection can be found even when $\Delta>0$. This effect will be analysed in detail in Section 3 .

The nonlinear correction can also strongly affect the size of the critical angle. When $\Delta \gg 2 \kappa \eta_{0}^{2},(9)$ gives $\tan \left(\theta_{c}\right) \approx(\Delta /(1-\Delta))^{1 / 2}$, which is the value corresponding to a linear plane wave at the interface. This is plotted with a dash-dotted line in figure $2(\mathrm{~b})$ that lies very close to the results for optical solitons in the $\Delta \gg 2 \kappa \eta_{0}^{2}$ regime (solid line). Nevertheless, as $\Delta \sim 2 \kappa \eta_{0}^{2}, \theta_{c}$ undergoes large changes, as is shown by the dashed line of figure 2(b). The generalised Snell's law predicts that interfaces showing linear external refraction $(\Delta<0)$ can also exhibit total internal reflection. This phenomenon is studied in Section 4. 
(a)

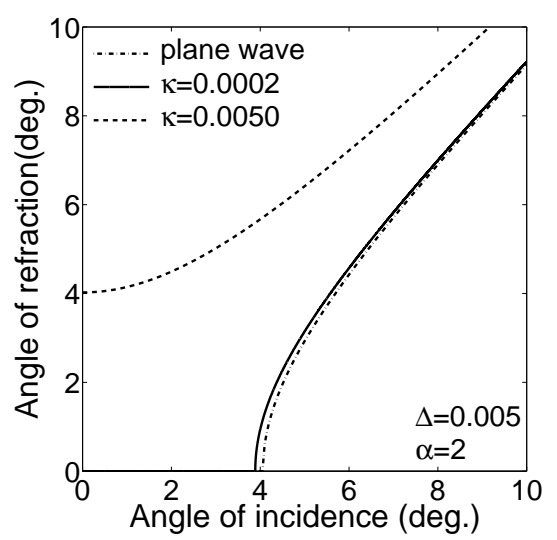

(b)

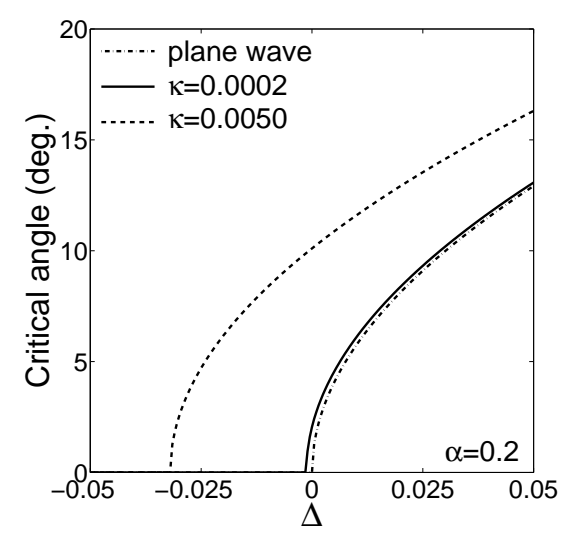

Figure 2. (a) Generalised Snell's law, and (b) critical angle, for different values of $\kappa$ and $\eta_{0}=1$.

Parameters in figures 2(a) and (b) are chosen to illustrate the Helmholtz type of nonparaxiality we are addressing in this work. Thus, we restrict our analyses to broad beams of low or moderate intensity $\kappa \eta_{0}^{2} \ll 1$ and values of $\Delta \sim 2 \kappa \eta_{0}^{2}$ for which nonlinearity significantly affects soliton refraction. Even though results shown in figures 2 (a) and (b) are restricted to angles smaller than $20^{\circ}$, for which the values of $\Delta$ used introduce relevant changes, the validity of the generalised Snell's law extends to arbitrary angles.

\section{Nonlinear external refraction}

We have seen how the refraction of optical solitons at an interface can be substantially affected by the nonlinear terms in the refractive indexes. These effects can be quantified by a mismatch parameter

$$
\delta=\Delta+2 \kappa \eta_{0}^{2}(1-\alpha)
$$

which captures both the linear and nonlinear contributions to the refractive index step across the boundary. Parameter $\delta$ alone can be used to predict how the soliton refracts at the interface: $\theta_{t}>\theta_{i}$ (external refraction) for $\delta<0, \theta_{t}<\theta_{i}$ (internal refraction) for $\delta>0$ and $\theta_{t}=\theta_{i}$ for $\delta=0$. This last case is a total transparency condition [37], obtained when the linear and nonlinear refractive index mismatches cancel each other. Under this condition, the soliton crosses the interface at a constant angle.

We now consider the particular case of $\Delta>0$. For linear interfaces, one would find internally refracting plane waves. If the nonlinear correction is such that $\delta<0$, nonlinear external refraction of optical solitons is found, as shown by the results for $\kappa=0.005$ in figure 2(a). Figure 3 illustrates this effect. In figure 3(a), lines correspond to the predictions from the generalised Snell's law (7) and points are from the numerical integration of the NLH equation [31]. For $\kappa=10^{-4}$, one obtains $\delta \sim \Delta$ and the angle of refraction (solid line) remains close to that expected for linear interfaces. However, 
as the nonparaxiality parameter is increased to $\kappa=2.5 \times 10^{-3}$ (giving $\delta=-0.003$ ), nonlinear external reflection is found (dashed line). In both cases, numerical results show very good agreement with the predictions of the generalised Snell's law.

(a)

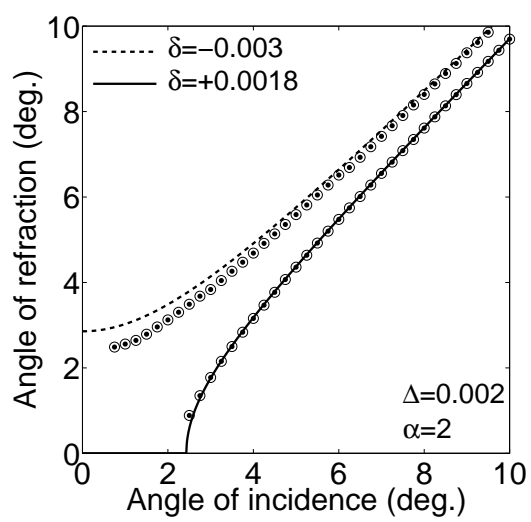

(b)

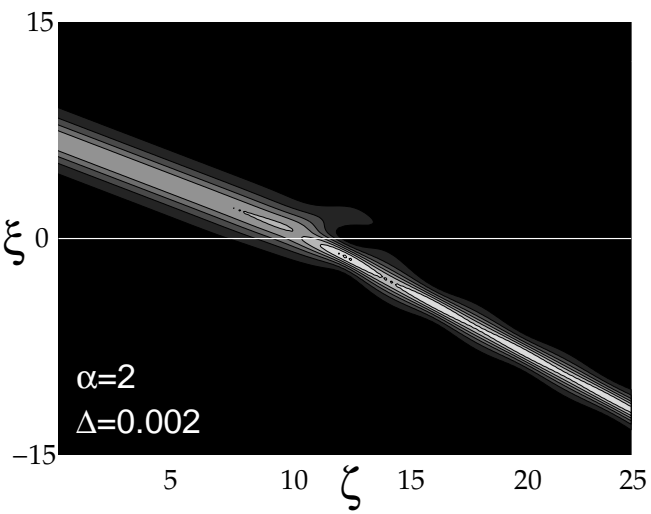

Figure 3. (a) Nonlinear external refraction $(\delta=-0.003)$ for $\Delta>0$ interfaces. (b) A Helmholtz soliton undergoes nonlinear external reflection at the same interface described in (a) when $\delta=-0.003$.

The NBPM [31] has been used to obtain the intensity plot of figure 3(b) which displays the evolution of a Helmholtz soliton impinging on the interface described in figure $3(\mathrm{a})$ when $\delta=-3 \times 10^{-3}$. After entering the second medium, the angle of refraction exceeds the angle of incidence since the conditions for nonlinear external refraction are satisfied. The soliton width decreases when entering the second medium due to the larger Kerr nonlinearity.

\section{Total internal reflection for $\Delta<0$ interfaces}

Interfaces exhibiting linear external refraction, $\Delta<0$, are most likely to pose intrinsically nonparaxial regimes due to the large angles of refraction typically found. Not surprisingly, previous NLS analyses have avoided this type of nonlinear boundary. The nonlinear Helmholtz generalisation of Snell's law predicts the new effect of total internal reflection for $\Delta<0$ interfaces.

The condition for total internal reflection at interfaces exhibiting linear external refraction requires that $\alpha<1$. This relationship between the Kerr coefficients of two adjoining media can inhibit soliton formation in the second medium if beam diffraction is not fully compensated by self focusing. In that case, the beam in the second medium undergoes diffractive spreading. Nonlinear-linear interfaces characterised by $\alpha=0$, constitute an extreme limit of $\alpha<1$ and are the most suitable to exhibit total internal 
reflection whenever $\Delta<0$. For these cases,

$$
\left.\tan \left(\theta_{c}\right)\right|_{\alpha=0}=\left(\frac{\Delta+2 \kappa \eta_{0}^{2}}{1-\Delta}\right)^{1 / 2}
$$

(a)

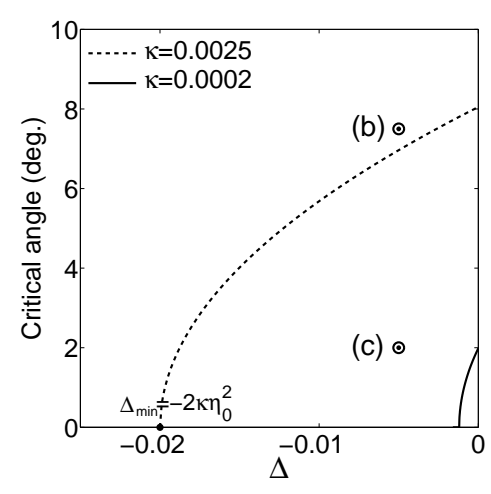

(b)

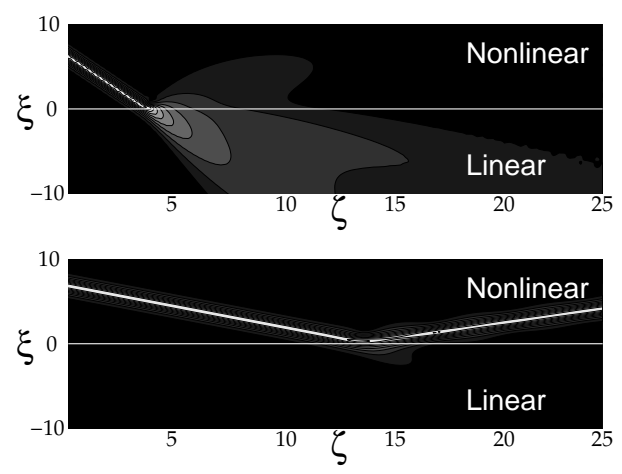

(c)

Figure 4. (a) Critical angle for $\Delta<0$ interfaces. Evolution of a Helmholtz soliton $\left(\eta_{0}=2\right)$ at a nonllinear-linear interface $(\Delta=-0.005)$ for: (b) $\theta_{i}>\theta_{c}$, and (c) $\theta_{i}<\theta_{c}$.

In figure 4(a), the critical angle is shown for two different values of $2 \kappa \eta_{0}^{2}$. The dashed line represents $\theta_{c}$ when $2 \kappa \eta_{0}^{2}=2 \times 10^{-2}$, showing that critical angles are obtained whenever the nonlinear index mismatch prevails over $\Delta$. In that case, the minimum value of $\Delta$ accommodating total internal reflection is $\Delta_{\text {min }}=-2 \kappa \eta_{0}^{2}$. When $\kappa=2 \times 10^{-4}$, the nonlinearity barely compensates the negative value of $\Delta$ (solid line) and the existence region for the critical angle is greatly reduced.

The two points marked as (b) and (c) in figure 4(a) correspond to the two surface plots on the right. These plots have been obtained from the numerical integration of the NLH [31] and show the behaviour of a soliton with $2 \kappa \eta_{0}^{2}=2 \times 10^{-2}$ impinging on a nonlinear-linear interface with $\Delta=-0.005$. As predicted in figure 4(b), a soliton propagating with an angle $\theta_{i}>\theta_{c}$ experiences diffractive spreading when it crosses the interface and enters the linear medium. On the other hand, for $\theta_{i}<\theta_{c}$ the soliton undergoes total internal reflection at the interface and is directed back into the nonlinear medium, as is shown in figure $4(\mathrm{c})$.

\section{Conclusions}

We have presented a study of nonlinear interfaces, separating two Kerr focusing media, based on the NLH equation. Our analyses preserve the full angular content of the problem, and hence overcome the limitations embedded in the paraxial approximation.

Two effects taking place within intrinsically nonparaxial regimes have been reported and analysed. These are nonlinear external refraction and total internal reflection for interfaces exhibiting linear internal and external refraction, respectively. Both 
effects have been predicted using a generalised Snell's law for Helmholtz solitons and numerically investigated by full integration of the NLH equation. Excellent agreement has been found between analytical predictions and numerical results.

\section{Acknowledgements}

This work has been funded by the Spanish MICINN and FEDER, Project number TEC2007-67429-C02-01 and JCyL, Project VA001A08.

\section{References}

[1] Kaplan A E 1977 Sov. Phys. JETP 45896

[2] Smith P W, Hermann J-P, Tomlinson W J and Maloney P J 1979 Appl. Phys. Lett. 35846

[3] Marcuse D 1980 Appl. Opt 193130

[4] Tomlinson W J, Gordon J P, Smith P W and Kaplan A E 1982 Appl. Opt. 212041

[5] Nesterov L A 1988 Opt. Spektrosk. 641166 [Opt. Spectrosc. (USSR) 64 694]

[6] Aceves A B, Moloney J V and Newell A C 1989 Phys. Rev. A 391809

[7] A.B. Aceves, J.V. Moloney and A.C. Newell 1989 Phys. Rev. A 39, 1828

[8] Aceves A B, Varatharajah P, Newell A C, Wright E M, Stegeman G I, Heatley D R, Moloney J V and Adachihara H 1990 J. Opt. Soc. Am. B 7963

[9] Varatharajah P, Newell A C, Moloney J V and Aceves A B 1990 Phys. Rev. A 421767

[10] Kivshar Yu S, Kosevich A M and Chubykalo O A 1990 Phys. Rev. A 411677

[11] Aliev Yu M, Boardman A D, Xie K and Zharov A A 1994 Phys. Rev. E 491624

[12] Aliev Yu M, Boardman A D, Smirnov A I, Xie K and Zharov A A 1996 Phys. Rev. E 535409

[13] Boardman A D, Bontemps P, Ilecki W and Zharov A A 2000 J. Mod. Opt. 471941

[14] Shadrivov I V and Zharov A A 2002 J. Opt. Soc. Am. B 19596

[15] Zakharov V E and Shabat A B 1972 Sov. Phys. JETP 3462

[16] Akhmediev N, Ankiewicz A and Soto-Crespo J M 1993 Opt. Lett. 18411

[17] Chi S and Guo Q 1995 Opt. Lett. 201598

[18] Crosignani B, Porto P D and Yariv A 1997 Opt. Lett. 22778

[19] Ciattoni A, Porto P D, Crosignani B and Yariv A 2000 J. Opt. Soc. Am. B 17809

[20] Crosignani B, Yariv A and Mookherjea S 2004 Opt. Lett. 291254

[21] Ciattoni A, Crosignani B, Mookherjea S and Yariv A 2005 Opt. Lett. 30516

[22] Ciattoni A, Crosignani B, Porto P D and Yariv A 2005 J. Opt. Soc. Am. B 221384

[23] G. Fibich 1996 Phys. Rev. Lett. 764356

[24] Chamorro-Posada P, McDonald G S and New G H C 1998 J. Mod. Opt. 451111

[25] Chamorro-Posada P, McDonald G S and New G H C 2000 J. Mod. Opt. 471877

[26] Chamorro-Posada P, McDonald G S and New G H C 2002 J. Opt. Soc. Am. B 191216

[27] Chamorro-Posada P and McDonald G S 2003 Opt. Lett. 28825

[28] Christian J M, McDonald G S, Potton R and Chamorro-Posada P 2006 Phys. Rev. E 74066612

[29] Christian J M, McDonald G S and Chamorro-Posada P 2007 J. Phys. A 401545

[30] Christian J M, McDonald G S and Chamorro-Posada P 2007 Phys Rev. A 76033833

[31] Chamorro-Posada P and McDonald G S 2006 Phys. Rev. E 74036609

[32] Ferrando A, Zacarés M, de Córdoba P -F, Binosi D and Montero A 2005 Phys. Rev. E 71016601

[33] Fibich G and Tsynkov S 2001 J. Comp. Phys. 171632

[34] Fibich G and Tsynkov S 2005 J. Comp. Phys. 210183

[35] Baruch G, Fibich G and Tsynkov S 2008 Opt. Expr. 1613323

[36] Chamorro-Posada P, McDonald G S and New G H C 2001 Opt. Comm. 1921

[37] Sánchez-Curto J, Chamorro-Posada P and McDonald G S 2007 Opt. Lett. 321126 